\title{
Hypotension, acidosis and vasodilation syndrome after heart transplant: incidence, risk factors, and prognosis
}

\author{
M.J Paniagua, M.G Crespo-Leiro, J Muñiz, E Vázquez, T Tabuyo, M Castro-Orjales, S \\ Fojon, J.M López I.P Garrido, A Juffé, A Castro-Beiras
}

\begin{abstract}
Background. HAV syndrome, the combination of hypotension, acidosis and vasodilation (HAV), is a serious postoperative complication after heart transplantation (HT). Its etiology and prognosis are poorly understood. Aim. To determine the incidence and prognosis of post-HT HAV syndrome and examine possible risk factors. Methods. Retrospective examination of the records of 85 consecutive patients who underwent HT between December 1999 and June 2002 sought the HAV criteria: systolic BP <85 mm Hg plus $\mathrm{HCO}_{3}<19 \mathrm{mEq} / \mathrm{l}$ whole excluding cardiogenic, hypovolemic and septic shock. Donor variables included sex, age, weight, height, cause of death, time in ICU, and ischemic time; while recipient variables, sex, age, weight, height, etiology of cardiopathy, previous cardiopulmonary bypass surgery, preoperative amiodarone, $\beta$-blockers, catecholamines, mechanical ventilation or intra aortic balloon pump (IABP), RVP, time on waiting list, pump time, reoperations, polytransfusion, preoperative creatinine, GOT, GPT and GGT, induction with OKT3 or anti-CD25, bypass-to-HAV time, duration of catecholamine treatment, and 1 month survival after HT.

Results. The $11 \mathrm{HAV}$ cases (13\%) appeared between 1 and $72 \mathrm{~h}$ after HT (75\% in the first hour). Catecholamines were used for 1 to 6 days; control was achieved within $48 \mathrm{~h}$ in $58 \%$ of cases. Two HAV patients (18\%) died within the first month versus six non-HAV patients $(8.1 \%)(P=.275)$. Only polytransfusion showed more than a borderline value to predict HAV syndrome.

Conclusions. HAV syndrome has an incidence of $13 \%$ and a mortality of $18 \%$ within 1 month post-HT. The only likely risk factor is polytransfusion.
\end{abstract}

In Spain, the Survival Rate after heart transplantation (HT) is currently $76 \%$ at 1 year and $63 \%$ at 5 years. ${ }^{1}$ One early postoperative complication of $\mathrm{HT}$ is a syndrome characterized by hypotension, acidosis and vasodilation, hereinafter referred to as the HAV syndrome. This syndrome is not specific to HT, having been observed after a variety of other cardiac surgical procedures involving extracorporeal circulation. ${ }^{2,3}$ and 4 We know of only one other study devoted exclusively to HAV syndrome after HT. ${ }^{5}$

HAV syndrome can lead to shock, multiple organ failure and death. It is thought to arise through a general inflammatory response involving the activation and adhesion to endothelial cells of polymorphonuclear leukocytes, as well as the release of cytokines, other inflammatory mediators and cytotoxic substances including leukotrienes. ${ }^{2 \text { and } 3}$ It is treated with vasopressors. ${ }^{6}$ It is sometimes difficult to distinguish from, and sometimes coexists with, other conditions involving low cardiac output and shock after heart surgery, such as hypovolemic or cardiogenic shock. Its diagnosis therefore requires echocardiography (to rule out cardiac dysfunction and/or cardiac tamponade) and hemodynamic characterization (to exclude hypovolemia and cardiogenic shock and to confirm low peripheral resistance).

Chemmalakuzhy et $\mathrm{al}^{5}$ reported that among $54 \%$ of HT patients who develop HAV syndrome $33 \%$ die within the first postoperative month. They also observed than post-HT HAV syndrome was related to donor and recipient weight $>75 \mathrm{~kg}$, to an ischemia time $>180 \mathrm{~min}$, to pre-HT use of $\beta$-blockers, and to Caucasian race (vs Afro-American). Given the scarcity of information on this syndrome in the HT context, we undertook to study its incidence and prognosis, and association with donor and recipient variables as possible risk factors among HT patients in our center.

\section{Patients and methods}

We retrospectively studied the medical records of 85 consecutive HT patients operated between December 1999 and June 2002. HT had been performed using the bicava technique, using cardiopulmonary bypass with systemic hypothermia (range, $28{ }^{\circ} \mathrm{C}-30{ }^{\circ} \mathrm{C}$ ), cardioplegic hematic 
anterograde cold $\left(10^{\circ} \mathrm{C}\right)$ intermitent with hot reperfusion $\left(36^{\circ} \mathrm{C}\right)$. HAV syndrome was diagnosed if systolic arterial pressure was less than $85 \mathrm{~mm} \mathrm{Hg}$, serum bicarbonate concentration less than $19 \mathrm{mEq} / \mathrm{l}$, and cardiogenic and hypovolemic shock excluded. The seven donor variables were age, sex, weight, height, cause of death, length of ICU stay and ischemic time. The 25 recipient variables were age, sex, weight, height, type of cardiomyopathy, previous surgery involving cardiopulmonary bypass, pre-HT use of $\beta$-blockers, pre-HT use of amiodarone, RVP, time on HT waiting list, preoperative use of catecholamines, preoperative mechanical ventilation, preoperative intra-aortic balloon pump, pump time, reoperations, polytransfusion, preoperative serum variables (creatinine, GOT, GPT, GGT), induction of immunosuppression with OKT3 or anti-CD25 antibodies, time between cardiopulmonary bypass and onset of HAV syndrome, duration of treatment with catecholamines, and survival 1 month after HT.

Statistical analysis: Values of continuous variables were reported as means \pm standard deviations, and those of categorical variables as percentages. Hypotheses were tested using chi-squared, Mann-Whitney or Student's $t$ tests, depending on the type of variable and the nature of its distribution.

\section{Results}

Of the 85 patients studied, $11(13 \%)$ satisfied the criteria for HAV syndrome. In $75 \%$ of these cases the syndrome appeared within the first hour and in all cases within 72 hours. Amine treatment was required for between 1 and 6 days; in 58\% of cases the syndrome was controlled within 2 days. Accumulated mortality 1 month post-HT was $18 \%(2 / 11 ; 95 \%$ CI, ranges $2 \%-57 \%$ ) versus $8 \%(6 / 74)$ among non-HAV patients $(P=.275)$. Univariate analyses showed the only significant between-group difference to be polytransfusion, post-HT (HAV 55\%, non-HAV $15 \% ; P=.007$ ) and, with borderline statistical significance, pump time (HAV 143.3 \pm 41.8 , non-HAV $124.3 \pm 27.9 ; P=.053$ ).

\section{Discussion}

In this study of $85 \mathrm{HT}$ patients the incidence of HAV syndrome was $13 \%$, less than a quarter of the incidence reported by Chemmalakuzhy et al. ${ }^{5}$ Possible causes of this discrepancy may relate to our failure to perform in all of our patients, systemic vascular resistance values (as Chemmalakuzhy et al did, namely, 800 dyns $\cdot \mathrm{cm}^{-5}$ cutoff), or differences in the method of cardioplegia, the details of which are not supplied by Chemmalakuzhy et al.

Of the variables evaluated as possible risk factors, the only one with respect to which there was a statistically significant difference between the HAV and non-HAV groups was post-HT polytransfusion, which was not considered in the study of Chemmalakuzhy et al. Pump time was also longer for patients who developed HAV syndrome, with borderline statistical significance for the difference between the two groups.

$\beta$-Blockers can reduce the vasoconstrictive response to vasodilatory stimuli, and in the study of Chemmalakuzhy et al all five patients given $\beta$-blockers preoperatively (7\% of his total study group) developed HAV syndrome, versus only $51 \%$ of those who did not receive $\beta$-blockers. In our study, $26 \%$ of 85 patients received $\beta$-blockers, pre-HT, a value that was not statistically different between the two groups.

Another factor associated with HAV syndrome in the Chemmalakuzhy et al study, and which has been identified as an indicator of poor prognosis in several studies of this syndrome, is obesity. ${ }^{7}$ However, in the present study we observed no association of HAV with weight, height or body mass index, although our series included no patients with body mass indices indicative of obesity.

The accumulated mortality rate of $18 \%$ at 1 month post-HT among patients with HAV syndrome was not significantly greater than that of non-HAV patients, $8 \%$. In the Chemmalakuzhy et al study there was also a slightly but not significantly higher death rate among HAV patients, namely $33 \%$ versus $15 \%$. In both cases the absence of statistical significance in the difference between HAV and non-HAV groups may be due either to the small size of the groups or to multifactorial origins of early death after HT.

\section{Conclusions}

In our center the incidence of HAV after HT is $13 \%$, of whom $18 \%$ die within 1 month of transplant. The only risk factors identified are polytransfusion at HT and, possibly, lengthy pump times. 


\section{References}

1. L. Almenar-Bonet. Rev Esp Cardiol, 55 (2002), p. 1286.

2. P. Menasché. Curr Opin Cardyol, 10 (1995), p. 597.

3. S.P. Allen, M.H. Yacoub. Curr Opin Cardyol, 10 (1995), p. 605.

4. A.R. Tambur, J. Chemmalakuzhy, J. Short, et al. Transplant Proc, 33 (2001), p. 2960

5. J. Chemmalakuzhy, M.R. Costanzo, P. Meyer, et al. J Heart Lung Transplant, 20 (2001), p. 1075.

6. M. Argenziano, J.M. Chen, A.F. Choudhri, et al. J Thorac Cardiovasc Surg, 116 (1998), p. 973.

7. K.L. Grady, C. White-Williams, D. Naftel, et al. J Heart Lung Transplant, 18 (1999), p. 750. 